\title{
Polyethylene/Dibenzylidene Sorbitol: effect of com- position on space charge of polymeric insulation systems
}

\author{
G. Gherbaz*, G. Chen and A. S. Vaughan \\ School of Electronic and Computer Science, University of Southampton \\ Highfield, Southampton SO17 1BJ, UK \\ * e-mail: gg04r@ecs.soton.ac.uk
}

\begin{abstract}
This paper examines the effects of 1,3:2,4dibenzylidene sorbitol (DBS), a low-molecular organic molecule, on the space charge distribution in polyethylene. Morphological studies of the blends show the presence of a DBS network in the host system. Avrami analysis shows an increase in the nucleation density of polyethylene with the nucleating agent. Accumulation of space charge is one of the main causes of failure for high voltage cables and it depends on many factors such as additives, materials and treatment. In the present study we investigate the effects of different quantities of DBS in a blend of polyethylene. The space charge response during a stressing period and decay after removal of the applied electric field were observed. The results show that space charge accumulations are dependent on the concentration of the nucleating agent. In particular, the decay in a sample with $0.3 \%$ DBS indicates shallow traps, whilst samples containing 1 and $3 \%$ DBS show evidence of deep trap charges.
\end{abstract}

\section{INTRODUCTION}

Polymeric materials are widely used in electrical insulation and the morphology of the polymer appears to play an important role in increasing the breakdown strength of the insulator [1]. Space charge is believed to be one of the main causes that determines the breakdown under an applied electrical field. Studies have shown a significant increase in electrical strength in the presence of spherulites in the bulk. Other studies showed that the presence of additives can improve the dielectric properties of polymers [2].

In the last decade research has focused on the interaction between a polyolefin and dibenzylidene sorbitol (DBS). This molecule is a physical gel that creates a 3dimensional network, upon which the host polymer can crystallize. Studies have focused on the increase in nucleation sites, at low DBS content, which results in a reduced optical scattering of the blend [2]. However the electrical response of polymer loaded with nucleating agents has not been investigated fully [3].

Polyethylene is one of the most widely used polymers for electrical insulation purpose and an improved morphology might enhance electrical properties of the polymer. Re- cently a few studies have shown an improved space charge distribution of low density polyethylene (LDPE) containing $0.3 \%$ sorbitol [3]. This remarkable result has provided information on the effect of nanofibrils on the matrix. Some studies have compared space charge decay of composite and nanocomposite polymers. Nelson and $\mathrm{Hu}$ found a much faster decay in the presence of nanocomposite due to a more conductive interface [4]. Chen et al recently studied space charge accumulation of LLDPE loaded with different concentrations of alumina. They showed different charge traps and decay in all the samples. The nature of the charge was suggested to be ionisation from nanoparticles and injection from the electrodes.

In this study, we consider the effects of adding three different quantities of DBS $(0.3,1$ and $3 \mathrm{wt} \%)$ which forms a nanofibrilar network on the morphology of PE. We report on how the morphology and the presence of a polar network can affect the charge distribution under direct current conditions.

\section{EXPERIMENTAL}

\section{A. Sample Preparation}

The following materials were used in this work: the high density, linear polyethylene (LPE) Rigidex 160-25 from $\mathrm{BP}$; the low density, branched polyethylene (BPE) LD100BW from Exxon; the 1,3:2,4-dibenzylidene sorbitol was obtained from Milliken under the trade name of Millad 3905. The LPE and BPE were first melted, mixed and extruded to give a blend containing $20 \%$ LPE and $80 \%$ BPE. DBS was added to the polyethylene blend by dissolution in xylene followed by precipitation in hexane; blends of PE containing $0.3 \%, 1 \%$ and $3 \%$ DBS were prepared.

The above materials were characterized by differential scanning calorimetry (DSC), using a Perkin Elmer DSC7 running Pyris software. This instrument was routinely calibrated using high purity indium. The samples were melted at $200{ }^{\circ} \mathrm{C}$ and held at this temperature for $2 \mathrm{~min}$ in order to erase the previous thermal history. The samples were cooled at a rate of $100{ }^{\circ} \mathrm{C} / \mathrm{min}$ to the desired temperature and crystallized to completion. 
Specimens from microscopy were prepared using an RMC CR21/MT7 cryo-ultramicrotome and then etched using a $1 \% \mathrm{w} / \mathrm{v}$ solution of potassium permanganate in an acid mix composed of 5 parts of concentrated sulphuric acid to 2 parts dry phosphoric acid to 1 part water, and finally recovered using standard procedures [5]. Samples were sputter coated with gold and subsequently examined with a FEI Quanta FEG 600 environmental scanning electron microscope (SEM).

A Grasby-Specac hydraulic press was used to produce film samples of known thickness $(200 \mu \mathrm{m})$ for space charge measurements. Each sample was melted at a temperature of $170{ }^{\circ} \mathrm{C}$ for a period of $2 \mathrm{~min}$ and subsequently immersed in an oil bath at a temperature of $117^{\circ} \mathrm{C}$ for a period of $20 \mathrm{~min}$ to allow complete crystallisation of the blend. Finally, all samples were degassed in a vacuum oven overnight.

\section{B. PEA system}

Space charge distributions were measured using the pulse electro-acoustic (PEA) system. In this, the sample is inserted between the lower and upper electrodes and a pulse voltage is applied to the sample. Silicone oil was used to make a good acoustic contact between the sample and the electrodes. PEA technique works on the principle of acoustic pressure waves proportional to the charge layers based on Lorentz's Law [6]. These waves are converted into voltage signal by a piezoelectric transducer and then amplified and captured by an oscilloscope (see fig.1). A deconvolution technique was used to convert the signal $\mathrm{Vs}(\mathrm{t})$ into space charge information across the sample.

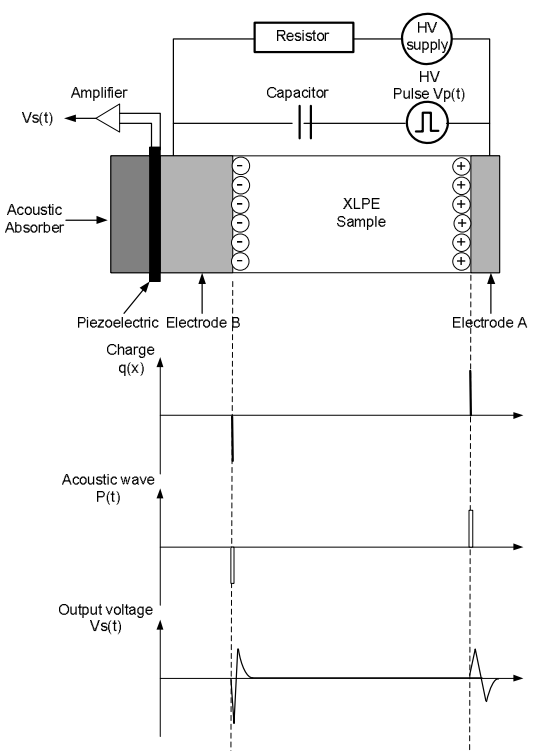

Fig. 1: Schematic diagram showing the principle of PEA system

\section{Voltage Profile}

Calibration was carried out at $2 \mathrm{kV}$. Space charge accumulations were conducted at $5 \mathrm{kV}$ for a period of $1 \mathrm{~h}$ at room temperature. After this time, the voltage was re- moved and the discharge process was recorded over $1 \mathrm{~h}$. During the charging phase, data were acquired every $30 \mathrm{~s}$ over the first $5 \mathrm{~min}$, and then subsequently every $5 \mathrm{~min}$ for the remaining time as shown in fig. 2.For a clearer interpretation of these results, data with voltage on and off were gathered during the charging process. The sample was short-circuited upon the removal of the external voltage and the charge decay was monitored for $1 \mathrm{~h}$. In this phase data were acquired every $5 \mathrm{~min}$. A typical voltage profile and the measurement points are shown in fig. 2 .

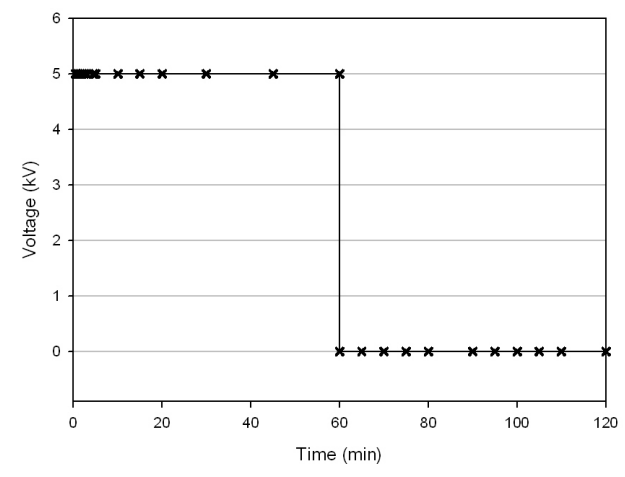

Fig. 2 Voltage profile at $5 \mathrm{kV}$ and PEA measurements points

\section{RESUlTS AND DisCUSSION}

\section{A. Growth Kinetics}

Standard Avrami analyses were applied to isothermal DSC data to provide the nucleation density data [4, 5]. A three dimensional growth rate constant, $K_{3}$, was derived according to Kowalewski and Galeski approximation:

$$
K_{3}=K_{e x p}^{3 / n}
$$

Fig. 3 shows the result obtained from $K_{3}$ values plotted as a function of crystallisation temperature for each system containing different DBS contents. It is apparent that in the presence of DBS, there is an increase in the growth rate which implies a modification in the nucleation and growth of the polymer blend. This result confirms an effective role of the nucleating agent as seen in other works as reported previously by Vaughan and Hosier [7].

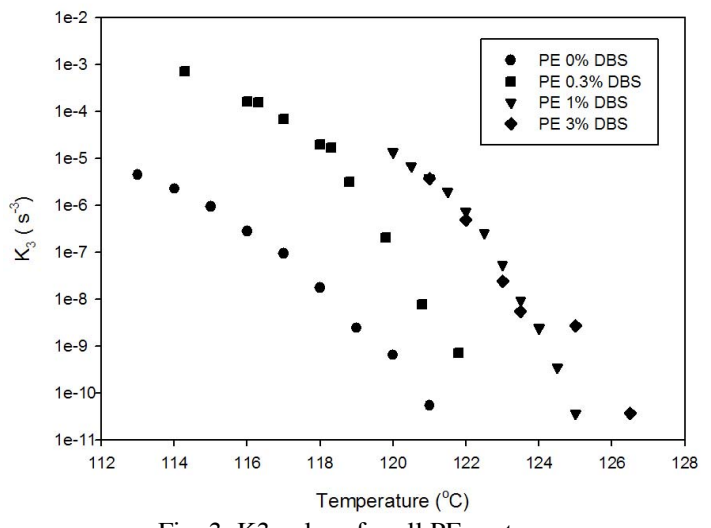

Fig. 3: K3 values for all PE systems. 


\section{B. Morphology}

Fig. 4 shows the morphology of a sample without DBS crystallized at $117{ }^{\circ} \mathrm{C}$. The presence of banded spherulites is evident throughout the sample. Similar results was found in the case of $0.3 \%$ DBS. Further addition of DBS modifies extensively the microstructure of polyethylene. Fig. 5 shows a sample containing $1 \%$ DBS crystallized at $117{ }^{\circ} \mathrm{C}$. The morphology is composed of polymer lamellar crystallized around DBS fibrils. The diameter of the fibrils is of the order of $100 \mathrm{~nm}$. The same type of microstructure was observed in the case of $3 \%$ DBS.

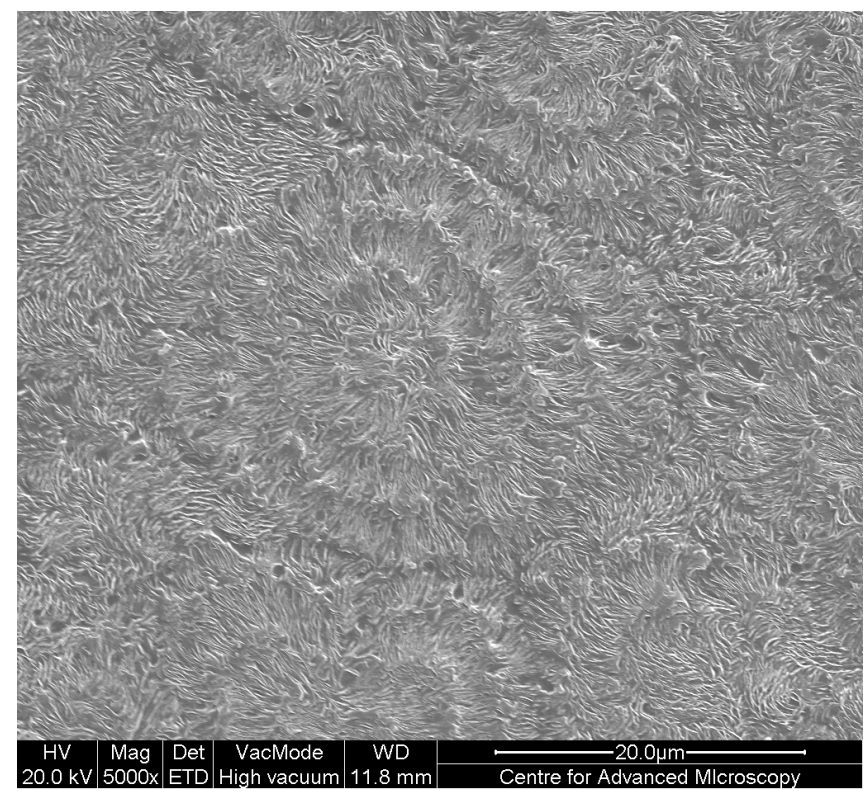

Fig. 4: PE 0\% DBS crystallized at $117^{\circ} \mathrm{C}$. Presence of spherulites

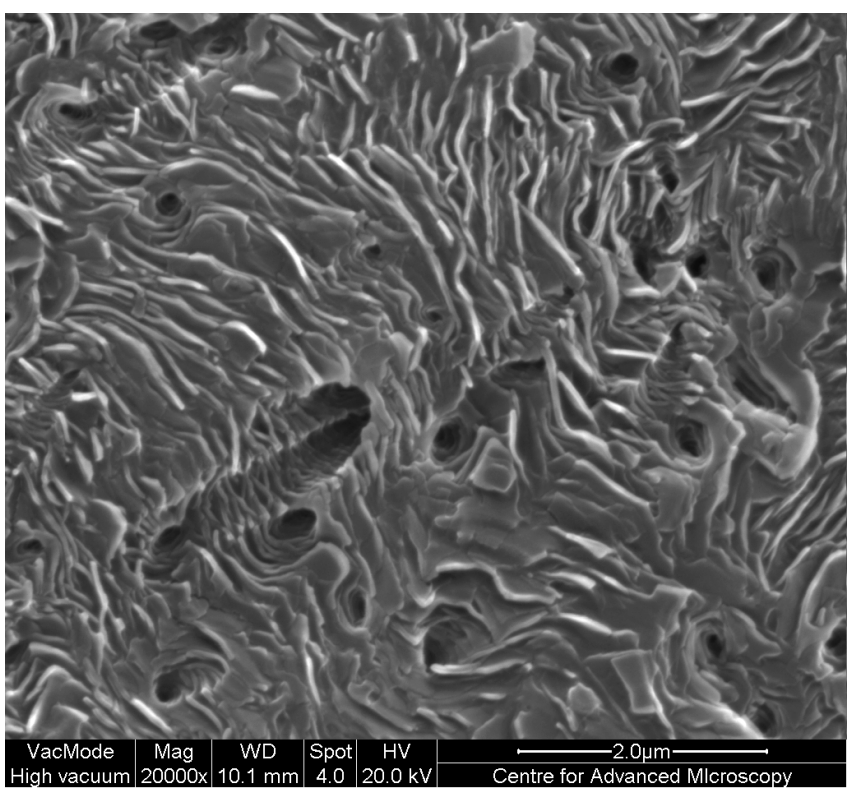

Fig. 5: PE $1 \%$ DBS crystallized at $117^{\circ} \mathrm{C}$. Polyethylene lamellae crystallised around bundles of DBS fibrils.

\section{Space Charge}

Fig. 6(a) shows the space charge formation during the voltage application period and only the space charge from the volts-off measurements are shown here. It can be clearly seen an increase of heterocharge close to the cathode and they are in agreement with previously reported data. Fig. 6(b) represents the decay of charge of the same sample over a period of $1 \mathrm{~h}$. A decrease of the charge can be noted near the cathode but there is still a presence of charge in the bulk after $1 \mathrm{~h}$

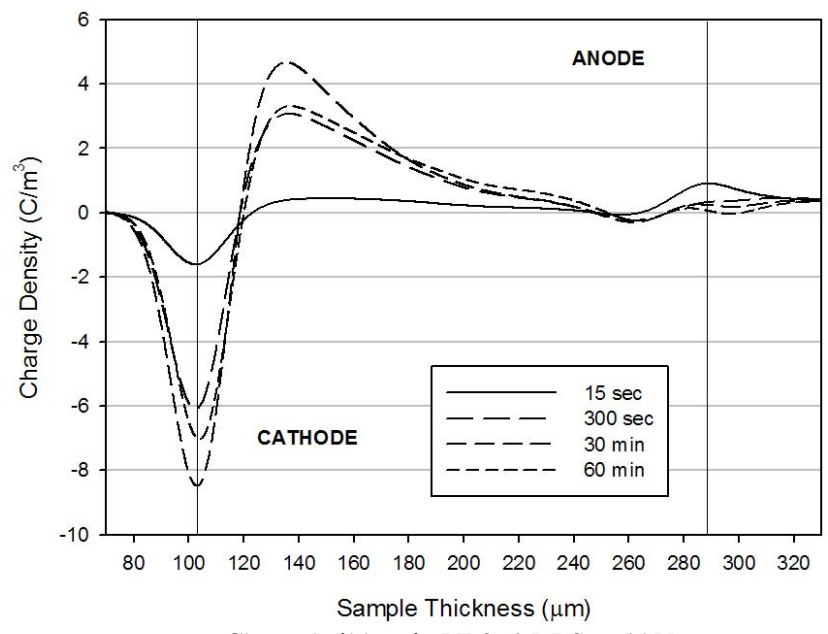

(a) Charge build-up in PE $0 \%$ DBS at $5 \mathrm{kV}$

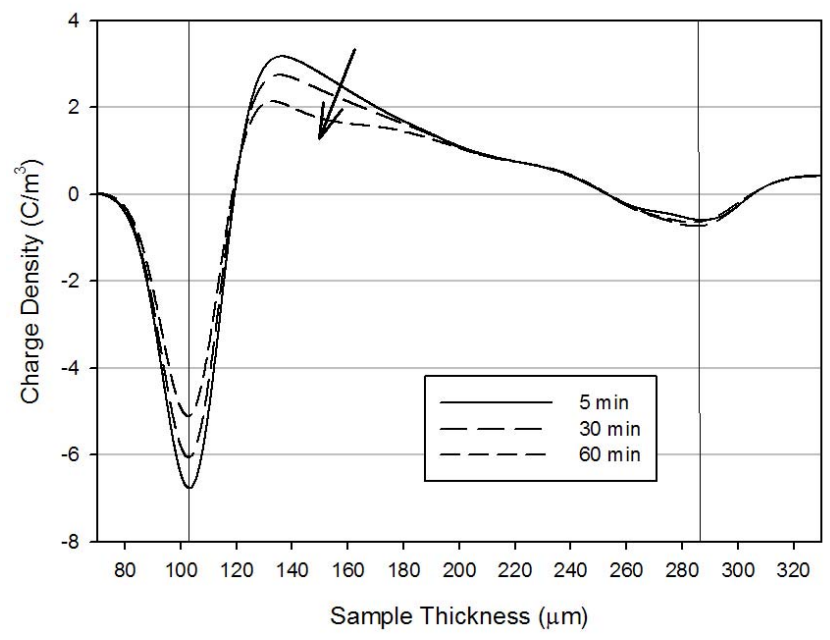

(b) Charge decay of PE $0 \%$ DBS performed in $1 \mathrm{~h}$ time

Fig. 6: Space charge measurements in PE blend without DBS

For the sake of brevity results from charging period of samples containing DBS can not be shown here, however the 0.3 $\%$ DBS shows presence of heterocharge near the cathode, whilst homocharge is present in 1 and $3 \%$ DBS.

Fig. 7 compares the space charge behaviour of the three blends containing different amount of DBS taken during the decay period. This result show a faster discharge in the case of $0.3 \%$ DBS which can be attributed to the presence of DBS as found by Xiang et al. [3]. It can be concluded that the charge distribution is improved and the nucleating agent 
acts as site of shallow traps. However the charge decay occurs more slowly in the case of $1 \%$ DBS (fig. 7(b)). It can be observed that after an initial decrease of the charge near the electrode, the residual charge remains constant. This result suggests that deep charge traps are present in the bulk. The site of the charge can be attributed to the presence of the fibrilar network or to the change in the microstructure of the polyethylene. Fig. 7(c) represents the space charge decay for $3 \%$ DBS. It can be clearly seen almost no decay of charge, which it makes conclude in an enhanced number of trapped charges in the system due to the polarity of the fibrils.
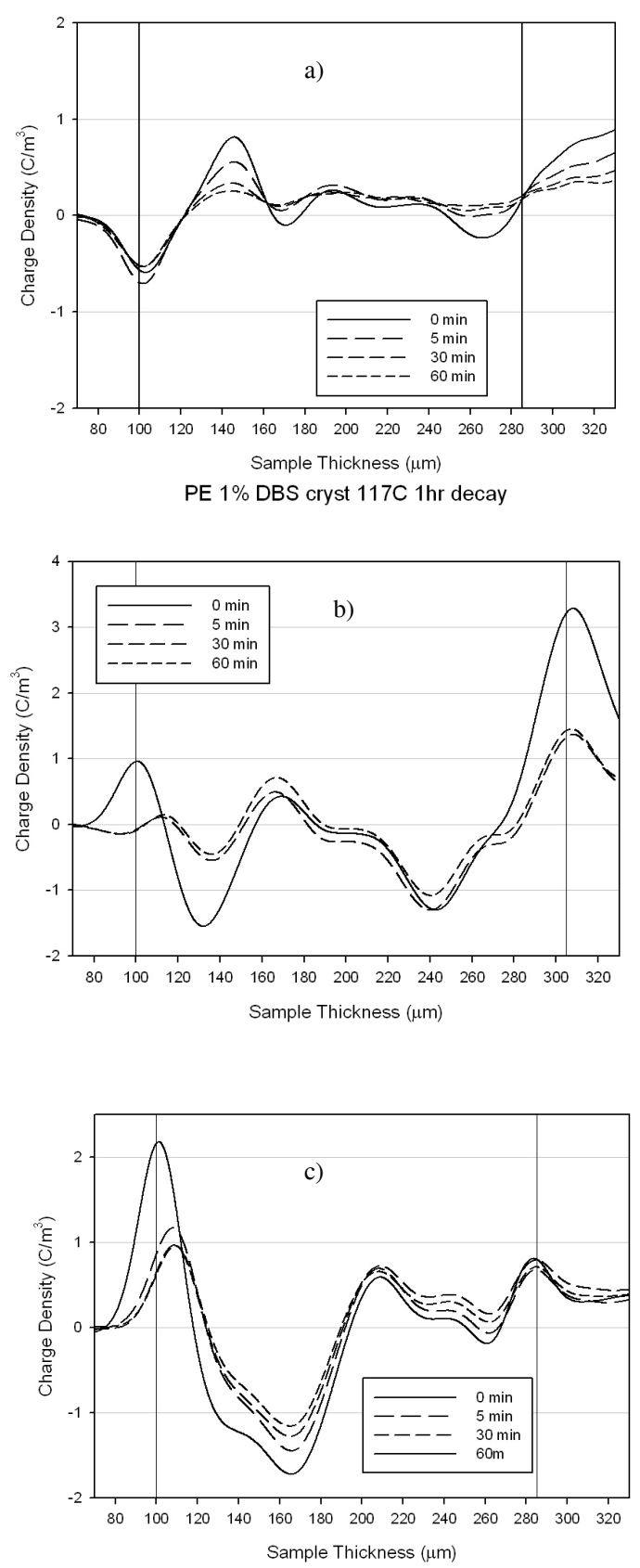

Fig. 7: (a) decay of PE $0.3 \%$ DBS; (b) PE $1 \%$ DBS; (c) PE $3 \%$ DBS.

\section{CONCLUSION}

The morphology and the space charge formation in PE blends loaded with different amounts of DBS has been investigated using DSC and the PEA technique at $5 \mathrm{kV}$.

DBS is an efficient nucleating agent at a concentration of $0.3 \%$ and above, where it forms a network within the polymer. However, the charge transport dynamics differ between $0.3 \%$ and $1 \%$, despite the fact that the polymer is, morphologically, equivalent and the DBS in both cases appears similarly disperse. The results reported have therefore imply that the local state of the DBS changes between a concentration of $0.3 \%$ and $1 \%$. .

\section{REFERENCES}

[1] I. L. Hosier, A. S. Vaughan and S. G. Swingler, "On the effects of morphology and composition on the electrical strength of polyethylene blends," $J$. Polym. Sci.: Part B: Polym. Phys., vol. 38, pp. 2309-2322, Sep. 2000.

[2] Y. Tanaka, G. Chen, Y. Zhao, A. Davies, A Vaughan, T Takada, "Effect of Additives on Morphology and Space Charge Accumulation in Low Density Polyethylene" IEEE Transactions on Dielectrics and Electrical Insultation, Vol. 10, pp. 148-153, 2003.

[3] Xiang L., Yi C., Qiangguo D, Yi Y., Demin T, "Charge distribution and crystalline structure in Polyethylene Nucleated with Sorbitol", J. Appl. Polym Sci, vol 82, pp. 611-619, 2001

[4] J. K. Nelson, Y. Hu, "Nanocomposite dielectrics - properties and implications", J. Phys. D-Applied Physics, vol. 38, 213-222, 2005.

[5] R. H. Olley and D. C. Bassett, "An Improved Permanganic Etchant for Polyolefines," Polymer, vol. 12, pp. 1707-1710, Dec. 1982.

[6] T. Maeno, T. Futami, H. Kushibe, T. Takada and C. M. Cooke, "Measurement of spatial charge distribution in thick dielectrics using the pulsed electroacustic method", IEEE Trans. On EI, vol. 23, pp. 433-439, 1988

[7] A. S. Vaughan and I. L. Hosier, "The effect of dibenzylidene sorbitol on the crystallization behaviour of polyethylene. J.l of Mater. Sci., 48 (8). pp. 2922 2928. 